\title{
Farmacogenética aplicada a cessação do tabagismo
}

\author{
Pharmacogenetics applied to smoking cessation \\ Farmacogenética aplicada a la cesación tabáquica
}

Recebido: 06/09/2021 | Revisado: 15/09/2021 | Aceito: 21/09/2021 | Publicado: 23/09/2021

\author{
Adriano Ferro Rotondano Filho \\ ORCID: https://orcid.org/0000-0002-8176-6432 \\ Universidade Evangélica de Goiás UniEVANGÉLICA, Brasil \\ E-mail: adrianoferro09@gmail.com \\ Thais Carolina Alves Cardoso \\ ORCID: https://orcid.org/0000-0002-0209-3840 \\ Universidade Evangélica de Goiás UniEVANGÉLICA, Brasil \\ E-mail: thaisscarolinaa@ hotmail.com \\ Jalsi Tacon Arruda \\ ORCID: https://orcid.org/0000-0001-7091-4850 \\ Universidade Evangélica de Goiás UniEVANGÉLICA, Brasil \\ E-mail: jalsitacon@gmail.com
}

\begin{abstract}
Resumo
Muitos estudos buscam as bases da farmacogenética para elucidar a adição nicotínica. O tabagismo tem sido associado a vários polimorfismos genéticos. Esta revisão integrativa reúne informações da literatura atualizada com o objetivo de avaliar a relação da dificuldade de cessação do tabagismo com as alterações genéticas. Buscas foram feitas nas bases de dados: PubMed, SciELO, LILACS e Google Acadêmico. Os estudos indicam que mesmo com os esforços intensivos para o controle do comportamento tabágico a cessação definitiva do tabagismo geralmente só ocorre após várias tentativas e o número de recaídas é muito grande. Fatores genéticos contribuem para a dificuldade e o sucesso nos esforços de cessação do tabagismo. Contudo, o desafio ainda é compreender, em termos moleculares, como as variações genéticas conferem suscetibilidade a cessação do tabagismo. No futuro, ensaios clínicos mais robustos poderão individualizar o tipo, a dosagem e a duração do tratamento da dependência tabágica, conforme o genótipo de cada fumante, maximizando a eficácia do esquema proposto. Assim, identificar as variantes de risco em cada gene candidato, os polimorfismos genéticos capazes de determinar de que modo cada variante altera a função de proteínas codificadas são de fundamental importância para que se comprove de fato essa relação da farmacogenética com o tabagismo.
\end{abstract}

Palavras-chave: Abandono do uso de tabaco; Genética humana; Nicotina; Tabagismo; Transtorno por uso de tabaco.

\begin{abstract}
Many studies seek the basis of pharmacogenetics to elucidate nicotine addiction. Smoking has been associated with several genetic polymorphisms. This integrative review gathers information from the updated literature to assess the relationship between difficulty in smoking cessation and genetic alterations. Searches were made in the databases: PubMed, SciELO, LILACS and Academic Google. Studies indicate that even with intensive efforts to control smoking behavior, definitive smoking cessation usually only occurs after several attempts and the number of relapses is very high. Genetic factors contribute to the difficulty and success of smoking cessation efforts. However, the challenge is still to understand, in molecular terms, how genetic variations confer susceptibility to smoking cessation. In the future, more robust clinical trials will be able to individualize the type, dosage, and duration of treatment for tobacco dependence, according to the genotype of each smoker, maximizing the effectiveness of the proposed regimen. Thus, identifying the risk variants in each candidate gene, the genetic polymorphisms capable of determining how each variant alters the function of encoded proteins, are of fundamental importance to really prove this relationship between pharmacogenetics and smoking.
\end{abstract}

Keywords: Abandonment of tobacco use; Human genetics; Nicotine; Smoking; Tobacco use disorder.

\section{Resumen}

Muchos estudios buscan las bases de la farmacogenética para dilucidar la adicción a la nicotina. El tabaquismo se ha asociado con varios polimorfismos genéticos. Esta revisión integradora recoge información de la literatura actualizada con el fin de evaluar la relación entre la dificultad para dejar de fumar y las alteraciones genéticas. Se realizaron búsquedas en las bases de datos: PubMed, SciELO, LILACS y Academic Google. Los estudios indican que incluso con esfuerzos intensivos para controlar el comportamiento del tabaquismo, el abandono definitivo del tabaquismo generalmente solo ocurre después de varios intentos y el número de recaídas es muy alto. Los factores genéticos contribuyen a la dificultad y el éxito de los esfuerzos para dejar de fumar. Sin embargo, el desafío sigue siendo comprender, en términos moleculares, cómo las variaciones genéticas confieren susceptibilidad al abandono del hábito de fumar. En el futuro, ensayos clínicos más robustos podrán individualizar el tipo, la dosis y la duración del tratamiento 
para la dependencia del tabaco, según el genotipo de cada fumador, maximizando la efectividad del régimen propuesto. Así, identificar las variantes de riesgo en cada gen candidato, los polimorfismos genéticos capaces de determinar cómo cada variante altera la función de las proteínas codificadas, son de fundamental importancia para probar realmente esta relación entre farmacogenética y tabaquismo.

Palabras clave: Abandono del consumo de tabaco; Genética humana; Nicotina; Tabaquismo; Trastorno por consumo de tabaco.

\section{Introdução}

Do tabaco (Nicotiana tabacum é a espécie mais consumida) é extraída a nicotina, um alcaloide que em cerca de 9 segundos após inalada atinge o cérebro e inicia ação estimulante ativando os receptores nicotínicos de acetilcolina (colinérgicos) (nAChRs), localizados nos receptores pré-sinápticos dos terminais axônicos dos gânglios do sistema nervoso autônomo, junção neuromuscular e sistema nervoso central, mas também em sítios somatodendríticos, axonais e pós-sinápticos (Silva, 2020). Indícios históricos indicam que seu uso surgiu aproximadamente no ano 1000 a.C., nas sociedades indígenas da América Central, nas proximidades da cidade de Tobaco, região de Yucatán. Em 1520 os colonizadores espanhóis viram pela primeira vez a planta sendo usada pelos índios da dessa região. Relatos ainda indicam que a planta "tabaco" teria sido denominada pelos colonizadores em homenagem a localidade onde a encontraram pela primeira vez (Cardoso et al., 2021a).

Existem mais de um bilhão de fumantes no mundo e 80\% deles vivem em 24 países, sendo dois terços em países de baixa e média renda onde a carga das doenças e mortes relacionadas ao tabaco é mais pesada. Estima-se que os fumantes atuais consumam cerca de seis trilhões de cigarros todos os anos (WHO, 2020). O cigarro possui mais de 4.800 substâncias tóxicas que provocam inúmeras morbidades, contudo, a dependência química expõe o tabagista as mais diversas vulnerabilidades gerando agravos que evoluem para doenças crônicas, como câncer, doenças cardiovasculares e pulmonares (Rodrigues et al., 2020).

O tabagismo é um dos mais importantes problemas em saúde pública. Apesar dos mais de 50 anos passados desde o primeiro documento sobre os prejuízos do fumo à saúde, o hábito de fumar ainda persiste como uma das principais causas preveníveis de morte no mundo (Santos, 2011; Cardoso et al., 2021b). Esse hábito apresenta uma evolução crônica e mata mais de 8 milhões de pessoas por ano, portanto o ato de fumar gera extenso comprometimento à saúde do indivíduo, tornando-o mais susceptível, por exemplo, a infecções (Silva et al., 2020).

Apesar de todos os malefícios do tabagismo a saúde ainda há pessoas que iniciam ou persistem nesse vício. Existem vários fatores que levam as pessoas ao hábito do fumo, como: curiosidade pelo produto, influência da publicidade maciça do cigarro nos meios de comunicação, que têm forte influência no comportamento tanto dos jovens como dos adultos; pais, professores, ídolos e amigos que também exercem uma grande influência levando a uma imitação de comportamento do adulto; necessidade de autoafirmação visto que os jovens iniciam o tabagismo antes dos 19 anos, momento da construção de sua personalidade; facilidade de aquisição da droga pelo baixo preço, problemas psiquiátricos como a depressão e esquizofrenia, e até mesmo a hereditariedade (Santos, 2011).

Diante dessa situação epidemiológica, a genética poderia explicar a grande variabilidade fenotípica dos indivíduos perante o hábito de fumar. O comportamento tabágico (iniciação do hábito, manutenção e cessação) é bastante variado quanto a sensibilização ou tolerância a nicotina, quanto à capacidade orgânica da metabolização, ao grau de intensidade da nicotinadependência, a maior ou menor dificuldade de abandonar seu uso, ao momento mais precoce ou não de início, aos riscos de recaídas, entre muitas outras peculiaridades. Essa grande variabilidade fenotípica deve-se, possivelmente, aos polimorfismos genéticos implicados nesse processo (Reichert et al., 2008).

Essa dependência explica por que cerca de $70 \%$ dos fumantes querem abandonar o fumo, mas não conseguem. Destes, cerca de um terço tem êxito por apenas um dia e menos de 10\% ficam em abstinência por cerca de 12 meses (Jha \& Chaloupka, 2000). A cessação definitiva do tabagismo geralmente só ocorre após várias tentativas e o número de recaídas é muito grande. 
O percentual em que isso ocorre é semelhante em quase todos os extratos sociais, inclusive nos indivíduos mais informados sobre as doenças relacionadas ao tabaco, como os profissionais de saúde (Cardoso et al., 2021b).

Por outro lado, é interessante observar que apenas uma parcela dos fumantes desenvolve tal dependência. Uma das grandes questões no estudo do tabagismo e de outras dependências na atualidade, é entender por que pessoas expostas a drogas se tornam ou não aditas a elas e o porquê nem todos os fumantes apresentam a mesma evolução (Meirelles, 2009). Desse modo, o tabagismo é um comportamento complexo multifatorial, em que as contribuições genética e ambiental são determinantes e significativos, tanto para a iniciação, como para a manutenção do hábito (Neale et al., 2005). Dessa forma, é importante o estudo do tema para que profissionais da saúde compreendam e individualizem o comportamento tabagista, até mesmo a duração do tratamento da dependência tabágica mediante o genótipo de cada fumante, maximizando assim a proficuidade da terapêutica proposta. Em vista disso, esse estudo tem como objetivo avaliar a relação da dificuldade de cessação do tabagismo com as alterações genéticas proporcionadas pelo hábito de fumar.

\section{Metodologia}

Foi realizada uma revisão integrativa da literatura a qual, através de uma busca específica e rigorosa na literatura, permite sintetizar as informações relevantes dos estudos encontrados relacionados ao tema em tese. Para tal, a questão norteadora foi "Há relação entre polimorfismos genéticos e a dificuldade na cessação do tabagismo?". Para responder a esse questionamento as buscas por artigos originais ou de revisão e relatos de casos, disponíveis completos que investigaram sobre essa temática, foram feitas nas bases de dados: PubMed, Scientific Electronic Library Online (SciELO), Google Acadêmico e Literatura LatinoAmericana e do Caribe em Ciências da Saúde (LILACS). Foram utilizados os seguintes descritores Ciências da Saúde (DeCS): "Hábito de fumar", "Genética Humana" e "Cessação do Hábito de Fumar" utilizados em português, inglês e espanhol, em combinação com auxílio dos operadores Booleanos (AND/OR). A coleta de dados foi realizada entre maio e julho de 2021. Os critérios de inclusão dos estudos foram: estudos publicados nos idiomas português, espanhol e inglês; sem restrição quanto a data de publicação, disponíveis na íntegra. Os critérios de exclusão foram: artigos de opinião, carta ao editor ou estudos nos quais os polimorfismos genéticos em relação ao hábito de fumar não fossem o objetivo, assim como outros trabalhos que não apresentavam correlação do tema e/ou com o objetivo pretendido, ou fora dos critérios descritos acima.

A primeira etapa de seleção foi identificar e retirar as duplicatas, após isso analisar os potenciais estudos e se preenchiam os critérios estabelecidos, passando por uma seleção inicial das publicações realizada com leitura do título e resumo para verificar se enquadravam na temática. Após essa etapa, os estudos pré-selecionados foram lidos na íntegra, empregando os critérios de inclusão e exclusão, para análise e obtenção das informações necessárias para a construção do presente estudo. Caso houvesse discordância entre os avaliadores sobre os critérios analisados, foi realizada discussão específica sobre o artigo em questão, confrontando ideias com base nos critérios adotados para a revisão. Ao final da seleção foram incluídos 37 estudos mais relevantes (Tabela 1). 
Tabela 1. Estudos encontrados nas buscas realizadas nas bases de dados.

\begin{tabular}{c|c|c}
\hline FONTES DE INFORMAÇÃO & ESTRATÉGIA DE BUSCA & RESULTADOS \\
\hline PubMed & $\begin{array}{c}\text { ("smoking cessation"[MeSH Terms] OR ("smoking"[All Fields] } \\
\text { AND "cessation"[All Fields]) OR "smoking cessation"[All Fields]) } \\
\text { AND ("human genetics"[MeSH Terms] OR ("human"[All Fields] } \\
\text { AND "genetics"[All Fields]) OR "human genetics"[All Fields] OR } \\
\text { ("human"[All Fields] AND "genetic"[All Fields]) OR "human } \\
\text { Lenetic"[All Fields]) AND ("smoke"[MeSH Terms] OR "smoke"[All } \\
\text { Fields] OR "smoke s"[All Fields] OR "smoked"[All Fields] OR } \\
\text { "smokes"[All Fields] OR "smoking"[MeSH Terms] OR } \\
\text { "smoking"[All Fields] OR "smokings"[All Fields] OR "smoking } \\
\text { s"[All Fields]) }\end{array}$ & $\begin{array}{c}557 \\
14\end{array}$ \\
Google Acadêmico & $\begin{array}{c}\text { Artigos selecionados incluídos na revisão: } \\
154.000\end{array}$ \\
\hline
\end{tabular}

Fonte: Autores (2021).

\section{Resultados e Discussão}

Estudos de expossoma avaliam o impacto de diversas exposições que um indivíduo sofre durante a vida (desde o desenvolvimento embrionário até a morte), uma medida cumulativa de influências ambientais e respostas biológicas associadas, incluindo exposições do ambiente ao qual está inserido, hábitos, além de processos endógenos sobre a saúde, mais especificamente sobre o componente genético (Vineis et al., 2020). Há 3 níveis de exposição: 1) Interna a qual compreende os processos do próprio corpo como o conteúdo celular - genético/bioquímico, metabolismo e estresse oxidativo, morfologia, microbiota intestinal e processos de envelhecimento etc. 2) A exposição externa específica que envolve desde o ambiente particular da criança na vida intrauterina estendendo-se ao ambiente familiar, irradiações, agentes infecciosos, contaminantes químicos, estilo de vida, uso de tabaco e álcool, atividade física, ocupação, dieta e intervenções médicas. 3) A exposição externa geral inclui as amplas exposições ao ambiente social e político como determinantes sociais da saúde e na vida financeira, o ambiente psicológico que envolve a educação, estresse e saúde mental, além dos ambientes urbanos/rurais e do próprio clima (Silva, 2020).

Os receptores colinérgicos (nAChRs) são ativados pelo neurotransmissor endógeno acetilcolina, organizados em um conjunto de 5 unidades de cadeias polipeptídicas, 9 isoformas Alfa ( $\alpha 2-\alpha 10)$, e 3 isoformas Beta ( $\beta 2-\beta 4)$, formando canais iônicos na membrana celular (Arinami et al., 2000). Devido à ampla distribuição a maioria dos neurotransmissores tem sua liberação estimulada após a administração da nicotina e a ação nos neuroreceptores é responsável pela alteração comportamental proporcionada pela droga. Quando a nicotina se liga a esses receptores, essa ligação promove alteração na configuração do receptor de membrana desencadeando a abertura do canal iônico com entrada de sódio ( $\mathrm{Na}+)$ ou cálcio $(\mathrm{Ca}++)$ que altera o potencial na membrana celular despolarizando, processo fundamental na transmissão do impulso nervoso através do axônio, resultando na liberação de neurotransmissores (Silva, 2020). O mais abundante e importante receptor é o $\alpha 4 \beta 2$ envolvido na dependência química a nicotina (constituído por 2 unidades $\alpha 4$ e 3 unidades $\beta 2$ ) (Markou, 2008).

A nicotina atua no sistema nervoso central através do sistema dopaminérgico mesolímbico, formado por neurônios que, a partir do mesencéfalo, fazem conexão com o sistema límbico através do núcleo accumbens, amígdala e córtex frontal (Silva, 2020). Estímulos nos receptores nAChRs nos corpos celulares e terminais dos neurônios dopaminérgicos da área tegmental ventral provocam a liberação de dopamina principalmente no núcleo accumbens e demais componentes do sistema límbico. Daí a resposta prazerosa do hábito de fumar (comportamento de reforço e recompensa), que intensifica a dependência química a nicotina e outras drogas (Benowitz, 2010).

Quando se estuda as questões genética, ambiental, fatores individuais (como o desenvolvimento neurológico, concomitância com eventuais doenças mentais), suas inter-relações e as respostas a determinadas drogas, essas apontam para o 
fato de que a contribuição genética possa ter um papel significativo no hábito tabágico, sendo possivelmente responsável por mais de $80 \%$ da variabilidade do risco de início da drogadição (Arinami et al., 2000; Volkow, 2005). Já a manutenção da dependência apresenta uma contribuição da hereditariedade de cerca de 67\% (Batra et al., 2003).

Os fatores genéticos representam um papel importante no comportamento individual em relação ao tabagismo, sendo essas influências mediadas, em parte, por diferenças biológicas e nos traços comportamentais, assim como nas respostas as propriedades da nicotina. Estas influências genéticas ocorrem no contexto de determinantes psicológicos e sociais, incluindo, entre outros, fatores como a depressão, gênero e a pressão ao indivíduo através da publicidade em relação ao uso do tabaco (Santos, 2011). Existem fatores hereditários envolvidos na iniciação ao tabagismo e na permanência na adição (Vink et al., 2005a). Li et al. (2003), em uma metanálise sobre parâmetros genéticos em relação a dependência nicotínica apontaram que a hereditariedade está relacionada em 37\% e 55\% (para homens e mulheres, respectivamente) na iniciação ao fumo e em 50\% e 46\% (para homens e mulheres, respectivamente) na permanência na adição. Estes dois fatores também se correlacionam de forma significativa com a quantidade de cigarros fumados por dia e a dependência nicotínica (Maes et al., 2004; Vink et al., 2005b).

Os fumantes tendem a ajustar o seu comportamento de modo a manter o nível de nicotina no cérebro constante. As diferentes formas de processamento da nicotina no sistema nervoso central podem influenciar o consumo e a necessidade de uma nova exposição. Além de constatarem que indivíduos com tolerância aumentada a nicotina, devido à alta capacidade de metabolizar a droga, podem experimentar poucas reações adversas em seu primeiro contato e, portanto, terão uma grande propensão a continuar fumando. Contrariamente, a baixa capacidade evidenciada em alguns indivíduos faz com que fumem menos e que uma menor porção de indivíduos se torne nicotino-dependente (Santos, 2011).

Os polimorfismos são variações genéticas que aparecem como consequência de mutações. A categoria mais básica é originada a partir de uma simples mutação, quando ocorre uma troca de uma única base nucleotídica por outra, chamado de polimorfismo de nucleotídeo único (SNP - Single Nucleotide Polymorphism), uma forma muito comum de variação genética nas sequências de DNA. Polimorfismos nos genes responsáveis pelo metabolismo da nicotina podem ser responsáveis pela diversidade entre os indivíduos em relação a tolerância a nicotina (Brazel et al., 2019). Os genes envolvidos no metabolismo da inativação da nicotina podem ser importantes no entendimento da dependência nicotínica (Chatkin, 2006). No grupo de enzimas citocromo P450 (CYP), em particular a enzima CYP2A6, ocorrem duas variantes do gene, CYP2A6*2 e CYP2A6*3, associadas a diminuição da atividade enzimática levando os indivíduos a serem catalogados como metabolizadores lentos da nicotina. Com isso, são possivelmente protetoras em relação a dependência do tabaco, já que os indivíduos com estas variantes fumam quantidades significativamente menores de cigarros por dia e apresentam maior probabilidade de parar de fumar quando comparados com indivíduos com a variante CYP2A6*1 (Carter et al., 2004).

A enzima CYP2B6 está envolvida no metabolismo da bupropiona (cloridrato de bupropiona) um inibidor seletivo da recaptação neuronal de catecolaminas (norepinefrina e dopamina), utilizada na terapêutica para cessação ao tabagismo. Portanto, variações neste genótipo podem ser preditivos no sucesso da cessação tabágica e indicativo para sucesso deste fármaco. Lee et al. (2007), verificaram que os fumantes que possuíam a variante CYP2B $6 * 6$ no genótipo foram os que obtiveram melhores resultados em relação a cessação do tabagismo quando tratados com bupropiona em comparação ao grupo placebo.

Li et al. (2003), identificaram em uma metanálise, 13 regiões cromossômicas que associam genética e tabagismo em pelo menos 2 amostras independentes. As regiões são nos cromossomos 3-7, 9-11, 17, 20, e 22. Dentre elas, as regiões nos cromossomos 9, 10, 11 e 17 foram as que tiveram valores mais significativos, mas os dados na literatura ainda são controversos devido ao tamanho amostral e etnias variarem entre os estudos. Regiões nos cromossomos 5, 8, 9, 17 e 20 foram associados com dependência nicotínica (Swan, 2006; Li et al., 2008; Han et al., 2010). Os cromossomos 5, 6, 14 e 22 foram associados com a iniciação ao tabagismo (Vink et al., 2004; Vink et al., 2006). Já os cromossomos 4, 5, 15 e 22 foram associados com o número de cigarros fumados ao dia (Berrettini et al., 2008; Swan et al., 2006; Li et al., 2008; Saccone et al., 2007). 
Estudos que associaram a genética com a cessação do tabagismo também foram conduzidos. O polimorfismo rs16969968 diminui as chances de sucesso na cessação da drogadição. Outro SNPs, rs3025343, localizado perto do gene da dopamina beta-hiroxilase ( $D B H$ - dopamine beta-hydroxylase) no cromossomo 9 foi associado com cessação do tabagismo (Bierut et al., 2007; Uhl et al., 2007; Thorgeirsson et al., 2008; Uhl et al., 2008; Tobacco and Genetics Consortium, 2010). O gene USH2A (Usher syndrome $2 A$ (autosomal recessive, mild)) localizado no cromossomo 1q41, demonstrou relação na cessação do tabagismo em um estudo de associação genômica ampla (GWAS - Genome-Wide Association Studies) e seleção genômica ampla (GWS - Genomic-Wide Selection) entre genética e tabagismo. Já o polimorfismo rs12126638 demonstrou associação em ser um fumante atual (Uhl et al., 2008; Vink et al., 2009).

Os SNPs em genes candidatos que contribuem no processo da abstinência de fumar bem-sucedida e até mesmo na cessação ao tabagismo, estão implicados em funções como: adesão celular, enzimas, reguladores transcricionais, neurotransmissores, receptores e regulação de DNA, RNA e proteínas (Uhl et al., 2007). Painéis de análise genética poderão ser produzidos, assim como os que já existem para análises de risco genético que avalia como a combinação de algumas informações genéticas contribui para seu risco de desenvolver determinadas doenças, como câncer. Novas observações replicadas fornecerão uma base cada vez mais forte para a compreensão dos mecanismos do hábito de fumar, ou seja, para identificar os indivíduos com maior ou menor probabilidade de sucesso nos esforços de cessação do tabagismo e para a adaptação de terapias para que os genótipos possam ajudar a combinar os fumantes com os tratamentos que apresentam maior probabilidade de benefícios (Cardoso et al., 2021b).

\section{Conclusão}

Fatores genéticos contribuem para a dificuldade e o sucesso nos esforços de cessação do tabagismo. É fundamental trabalhar com estudos que identifiquem quais são os genes relacionados com o uso do tabaco para que assim auxiliem os indivíduos na interrupção do hábito de fumar ou até mesmo para reduzir os agravos que os fumantes podem sofrer mediante esse vício. Entretanto, o desafio é de entender, em termos moleculares, como as variações genéticas conferem suscetibilidade à cessação do tabagismo. Diante disso, é preciso novos estudos que sejam capazes de identificar as verdadeiras variantes de risco em cada gene candidato, e determinar de que modo cada variante altera a função de cada proteína codificada. Por isso, mais estudos sobre polimorfismos genéticos em populações independentes são de fundamental importância para que se comprove de fato essa relação com o tabagismo.

\section{Referências}

Arinami, T., Ishiguro, H., \& Onaivi, E. S. (2000). Polymorphisms in genes involved in neurotransmission in relation to smoking. European journal of pharmacology, 410(2-3), 215-226. https://doi.org/10.1016/s0014-2999(00)00816-5

Batra, V., Patkar, A. A., Berrettini, W. H., Weinstein, S. P., \& Leone, F. T. (2003). The genetic determinants of smoking. Chest, 123(5), 1730-1739. https://doi.org/10.1378/chest.123.5.1730

Benowitz N. L. (2010). Nicotine addiction. The New England journal of medicine, 362(24), 2295-2303. https://doi.org/10.1056/NEJMra0809890

Berrettini, W., Yuan, X., Tozzi, F. Song, K., Francks, C., Chilcoat, H., \& Mooser, V. (2008). $\alpha-5 / \alpha-3$ nicotinic receptor subunit alleles increase risk for heavy smoking. Molecular psychiatry, 13(4), 368-373. https://doi.org/10.1038/sj.mp.4002154

Bierut, L. J., Madden, P. A., Breslau, N., Johnson, E. O., Hatsukami, D., Pomerleau, O. F., Swan, G. E., Rutter, J., Bertelsen, S., Fox, L., Fugman, D., Goate, A. M., Hinrichs, A. L., Konvicka, K., Martin, N. G., Montgomery, G. W., Saccone, N. L., Saccone, S. F., Wang, J. C., Chase, G. A., \& Ballinger, D. G. (2007). Novel genes identified in a high-density genome wide association study for nicotine dependence. Human molecular genetics, 16(1), 24-35. https://doi.org/10.1093/hmg/dd1441

Brazel, D. M., Jiang, Y., Hughey, J. M., Turcot, V., Zhan, X., Gong, J., Batini, C., Weissenkampen, J. D., Liu, M., CHD Exome+ Consortium, Consortium for Genetics of Smoking Behaviour, Barnes, D. R., Bertelsen, S., Chou, Y. L., Erzurumluoglu, A. M., Faul, J. D., Haessler, J., Hammerschlag, A. R., Hsu, C., Kapoor, M., \& Vrieze, S. (2019). Exome Chip Meta-analysis Fine Maps Causal Variants and Elucidates the Genetic Architecture of Rare Coding Variants in Smoking and Alcohol Use. Biological psychiatry, 85(11), 946-955. https://doi.org/10.1016/j.biopsych.2018.11.024 
Cardoso, T. C. A., Rotondano Filho, A. F., Dias, L. M., \& Arruda, J. T. (2021a). Aspectos associados ao tabagismo e os efeitos sobre a saúde. Research, Society and Development, 10(3), e11210312975-e11210312975.

Cardoso, T. C. A., Rotondano Filho, A. F., \& Arruda, J. T. (2021b). Correlação entre pandemias: Tabagismo e a COVID-19. Research, Society and Development, 10(10), e222101018442. https://doi.org/10.33448/rsd-v10i10.18442

Carter, B., Long, T., \& Cinciripini, P. (2004). A meta-analytic review of the CYP2A6 genotype and smoking behavior. Nicotine \& tobacco research: official journal of the Society for Research on Nicotine and Tobacco, 6(2), 221-227. https://doi.org/10.1080/14622200410001676387

Chatkin, J. M. (2006). The influence of genetics on nicotine dependence and the role of pharmacogenetics in treating the smoking habit. Jornal Brasileiro de Pneumologia, 32(6), 573-579. https://doi.org/10.1590/S1806-37132006000600016

Han, S., Gelernter, J., Luo, X., \& Yang, B. Z. (2010). Meta-analysis of 15 genome-wide linkage scans of smoking behavior. Biological psychiatry, 67(1), 1219. https://doi.org/10.1016/j.biopsych.2009.08.028

Jha, P., \& Chaloupka, F. J. (2000). Tobacco control in developing countries. Oxford University Press. 490 pp. Health Education Research, https://doi.org/10.1093/her/16.3.384

Lee, A. M., Jepson, C., Hoffmann, E., Epstein, L., Hawk, L. W., Lerman, C., \& Tyndale, R. F. (2007). CYP2B6 genotype alters abstinence rates in a bupropion smoking cessation trial. Biological psychiatry, 62(6), 635-641. https://doi.org/10.1016/j.biopsych.2006.10.005

Li, M. D., Cheng, R., Ma, J. Z., \& Swan, G. E. (2003). A meta-analysis of estimated genetic and environmental effects on smoking behavior in male and female adult twins. Addiction (Abingdon, England), 98(1), 23-31. https://doi.org/10.1046/j.1360-0443.2003.00295.x

Li, M. D., Ma, J. Z., Payne, T. J., Lou, X. Y., Zhang, D., Dupont, R. T., \& Elston, R. C. (2008). Genome-wide linkage scan for nicotine dependence in European Americans and its converging results with African Americans in the Mid-South Tobacco Family sample. Molecular psychiatry, 13(4), 407-416. https://doi.org/10.1038/sj.mp.4002038

Maes, H. H., Sullivan, P. F., Bulik, C. M., Neale, M. C., Prescott, C. A., Eaves, L. J., \& Kendler, K. S. (2004). A twin study of genetic and environmental influences on tobacco initiation, regular tobacco use and nicotine dependence. Psychological medicine, 34(7), 1251-1261. https://doi.org/10.1017/s0033291704002405

Markou A. (2008). Review. Neurobiology of nicotine dependence. Philosophical transactions of the Royal Society of London. Series B, Biological sciences, 363(1507), 3159-3168. https://doi.org/10.1098/rstb.2008.0095

Meirelles, R. H. S. (2009). Tabagismo e DPOC-dependência e doença-fato consumado. Pulmão RJ - Atualizações Temáticas, 1(1), 13-19.

Neale, B. M., Sullivan, P. F., \& Kendler, K. S. (2005). A genome scan of neuroticism in nicotine dependent smokers. American Journal of Medical Genetics Part B: Neuropsychiatric Genetics, 132(1), 65-69. https://doi.org/10.1002/ajmg.b.30095

Reichert, J., Araújo, A. J. D., Gonçalves, C. M. C., Godoy, I., Chatkin, J. M., Sales, M. D. P. U., \& Santos, S. R. R. D. A. (2008). Diretrizes para cessação do tabagismo-2008. Jornal Brasileiro de Pneumologia, 34(10), 845-880. https://doi.org/10.1590/S1806-37132008001000014

Rodrigues, L. A., Rodrigues, A. D. F. M., Patrício, J. A. L., \& Breda, I. S. (2020). A relação entre o tabagismo e a hospitalização por COVID-19: revisão integrativa. Unesc em Revista, 4(2), 99-113.

Saccone, S. F., Pergadia, M. L., Loukola, A., Broms, U., Montgomery, G. W., Wang, J. C., Agrawal, A., Dick, D. M., Heath, A. C., Todorov, A. A., Maunu, H., Heikkila, K., Morley, K. I., Rice, J. P., Todd, R. D., Kaprio, J., Peltonen, L., Martin, N. G., Goate, A. M., \& Madden, P. A. (2007). Genetic linkage to chromosome 22q12 for a heavy-smoking quantitative trait in two independent samples. American journal of human genetics, 80(5), 856-866. https://doi.org/10.1086/513703

Santos, V. A. (2011). Inter-relações entre tabagismo, sintomas depressivos e genética. Tese (Doutorado em Medicina e Ciências da Saúde, Área de concentração: Clínica Médica) - Pontifícia Universidade Católica do Rio Grande do Sul, Porto Alegre. 108 f. https://repositorio.pucrs.br/dspace/bit stream/10923/4318/1/000432105-Texto\%2BCompleto-0.pdf Acesso: 10/08/21

Silva, A. L. O. D., Moreira, J. C., \& Martins, S. R. (2020). COVID-19 e tabagismo: uma relação de risco. Cadernos de Saúde Pública, 36(5). https://doi.org/10.1590/0102-311X00072020

Silva, K. M. M. (2020). Tabagismo: avaliação da associação com experiências adversas na infância e polimorfismo genético em uma comunidade universitária brasileira. Tese (Doutorado, Programa de Pneumologia) Faculdade de Medicina da Universidade de São Paulo, São Paulo. 221 f. http://www.pgpneumologia.incor.usp.br/images/KEYLA_MEDEIROS_MAIA_SILVA.pdf

Swan, G. E., Hops, H., Wilhelmsen, K. C., Lessov-Schlaggar, C. N., Cheng, L. S., Hudmon, K. S., Amos, C. I., Feiler, H. S., Ring, H. Z., Andrews, J. A., Tildesley, E., \& Benowitz, N. (2006). A genome-wide screen for nicotine dependence susceptibility loci. American journal of medical genetics. Part B, Neuropsychiatric genetics: the official publication of the International Society of Psychiatric Genetics, 141B(4), 354-360. https://doi.org/10.1002/ajmg.b.30315

Thorgeirsson, T. E., Geller, F., Sulem, P., Rafnar, T., Wiste, A., Magnusson, K. P., Manolescu, A., Thorleifsson, G., Stefansson, H., Ingason, A., Stacey, S. N., Bergthorsson, J. T., Thorlacius, S., Gudmundsson, J., Jonsson, T., Jakobsdottir, M., Saemundsdottir, J., Olafsdottir, O., Gudmundsson, L. J., Bjornsdottir, G., \& Stefansson, K. (2008). A variant associated with nicotine dependence, lung cancer and peripheral arterial disease. Nature, 452(7187), 638-642. https://doi.org/10.1038/nature06846

Tobacco and Genetics Consortium (2010). Genome-wide meta-analyses identify multiple loci associated with smoking behavior. Nature genetics, 42(5), 441447. https://doi.org/10.1038/ng.571

Uhl, G. R., Liu, Q. R., Drgon, T., Johnson, C., Walther, D., \& Rose, J. E. (2007). Molecular genetics of nicotine dependence and abstinence: whole genome association using 520,000 SNPs. BMC genetics, 8(1), 10. https://doi.org/10.1186/1471-2156-8-10 
Research, Society and Development, v. 10, n. 12, e308101220279, 2021

(CC BY 4.0) | ISSN 2525-3409 | DOI: http://dx.doi.org/10.33448/rsd-v10i12.20279

Uhl, G. R., Liu, Q. R., Drgon, T., Johnson, C., Walther, D., Rose, J. E., David, S. P., Niaura, R., \& Lerman, C. (2008). Molecular genetics of successful smoking cessation: convergent genome-wide association study results. Archives of general psychiatry, 65(6), 683-693. https://doi.org/10.1001/archpsyc.65.6.683

Vineis, P., Robinson, O., Chadeau-Hyam, M., Dehghan, A., Mudway, I., \& Dagnino, S. (2020). What is new in the exposome?. Environment international, 143, 105887. https://doi.org/10.1016/j.envint.2020.105887

Vink, J. M., Beem, A. L., Posthuma, D., Neale, M. C., Willemsen, G., Kendler, K. S., Slagboom, P. E., \& Boomsma, D. I. (2004). Linkage analysis of smoking initiation and quantity in Dutch sibling pairs. The pharmacogenomics journal, 4(4), 274-282. https://doi.org/10.1038/sj.tpj.6500255

Vink, J. M., Posthuma, D., Neale, M. C., Eline Slagboom, P., \& Boomsma, D. I. (2006). Genome-wide linkage scan to identify Loci for age at first cigarette in Dutch sibling pairs. Behavior genetics, 36(1), 100-111. https://doi.org/10.1007/s10519-005-9012-0

Vink, J. M., Smit, A. B., de Geus, E. J., Sullivan, P., Willemsen, G., Hottenga, J. J., Smit, J. H., Hoogendijk, W. J., Zitman, F. G., Peltonen, L., Kaprio, J., Pedersen, N. L., Magnusson, P. K., Spector, T. D., Kyvik, K. O., Morley, K. I., Heath, A. C., Martin, N. G., Westendorp, R. G., Slagboom, P. E., ,.. Boomsma, D. I. (2009). Genome-wide association study of smoking initiation and current smoking. American journal of human genetics, 84(3), 367-379. https://doi.org/10.1016/j.ajhg.2009.02.001

Vink, J. M., Willemsen, G., \& Boomsma, D. I. (2005a). Heritability of smoking initiation and nicotine dependence. Behavior genetics, 35(4), 397-406. https://doi.org/10.1007/s10519-004-1327-8

Vink, J. M., Willemsen, G., Beem, A. L., \& Boomsma, D. I. (2005b). The Fagerström Test for Nicotine Dependence in a Dutch sample of daily smokers and ex-smokers. Addictive behaviors, 30(3), 575-579. https://doi.org/10.1016/j.addbeh.2004.05.023

Volkow N. D. (2005). What do we know about drug addiction? The American journal of psychiatry, 162(8), 1401-1402. https://doi.org/10.1176/appi.ajp.162.8.1401

WHO. World Health Organization. Tobacco. Genebra: WHO, 2020. https://www.who.int/news-room/fact-sheets/detail/tobacco. 